\title{
Predictors of Arterial Stiffness Amongst the 24- Hour Ambulatory Blood Pressure Variables in Hypertensive Patients
}

Vishal Bhandari ${ }^{1}$, Kamal Sharma ${ }^{2}$, Purva Shah ${ }^{3}$, Erum Khan ${ }^{3}$, Hardik D. Desai ${ }^{4}$, Tanisha Vora ${ }^{5}$, Sukriti Bhalla ${ }^{6}$, Dhruvkumar Gadhiya ${ }^{5}$, Manish Bansal ${ }^{7}$, Ravi R. Kasliwal ${ }^{8}$

1. Interventional Cardiology, Tagore Hospital and Heart Care Center, Jalandhar, IND 2. Cardiology Department, U.N. Mehta Institute of Cardiology and Research Centre, Ahmedabad, IND 3. Medicine, B.J. Medical College, Ahmedabad, IND 4. Internal Medicine, Gujarat Adani Institute of Medical Sciences, Krantiguru Shyamji Krishna Verma (KSKV) University, Bhuj, IND 5. Graduate Medical Education, Smt. Nathiba Hargovandas Lakhmichand (NHL) Municipal Medical College, Ahmedabad, IND 6. Cardiology, Akash Healthcare - Super Speciality Hospital, New Delhi, IND 7. Cardiology, Medanta - The Medicity, Gurgaon, IND 8. Cardiology, Medanta Hospital, Gurgaon, IND

Corresponding author: Kamal Sharma, drkamalsharma@unmicrc.org

\section{Abstract}

\section{Objective}

The objective of the study is to identify the predominant determinants of arterial stiffness as assessed by pulse-wave-velocity (PVW) amongst various 24-hour ambulatory blood pressure monitoring (ABPM) parameters in Indian hypertensive subjects.

\section{Method}

Subjects of both genders between 18-60 years with hypertension and who were either drug naïve or on stable anti-hypertensive treatment for at least three months were included in the study. All subjects underwent clinical evaluation with a medical history, biochemical investigations, and assessment of arterial stiffness by PWV along with 24-hour ABPM.

\section{Results}

We found the males were younger than females amongst hypertensive cohort $(41.53 \pm 10.89$ years vs. $52.2 \pm$ 5.17 years, respectively; $p=0.001$ ) and had shorter duration of hypertension ( $41.42 \pm 49.14$ months vs. $87.8 \pm$ 74.55 months, respectively; $\mathrm{p}=0.012$ ) and had lower 24 -hour average pulse pressure (aPP; $49.1 \pm 7.8 \mathrm{~mm} \mathrm{Hg}$ vs. $57.83 \pm 8.92 \mathrm{~mm} \mathrm{Hg}$, respectively; $\mathrm{p}=0.001$ ) at baseline. Younger people ( $<40$-years) as compared to those $>40$-years of age had the lower carotid-femoral (cf) PWV $(972.8 \pm 125.0 \mathrm{~cm} / \mathrm{sec}$ vs. $1165.0 \pm 208.4 \mathrm{~cm} / \mathrm{sec}$, respectively; $\mathrm{p}=0.001)$ and average brachial-ankle (ba) PWV $(1413.7 \pm 160.4 \mathrm{~cm} / \mathrm{sec}$ and $1640.0 \pm 227.1$ $\mathrm{cm} / \mathrm{sec}$, respectively; $\mathrm{p}=0.001$ ). Bivariate analysis revealed that amongst all the 24 -hour ABPM parameters, 24-hour aPP had the strongest correlation $(\mathrm{r}=0.414, \mathrm{p}=0.003)$ with arterial stiffness as assessed by PWV. Also, statistically significant correlation was found in age group $<40$ years between cf-PWV and both 24hour aPP $(\mathrm{r}=0.54, \mathrm{p}=0.025)$ as well as night-time aPP $(\mathrm{r}=0.59, \mathrm{p}=0.013)$

Review began $12 / 13 / 2020$ Review ended 12/16/2020 Published 12/21/2020

๑) Copyright 2020 Bhandari et al. This is an open access article distributed under the terms of the Creative Commons Attribution License CC-BY 4.0, which permits unrestricted use, distribution, and reproduction in any medium, provided the original author and source are credited.

\section{Conclusion}

We conclude that 24-hour aPP showed the strongest correlation with arterial stiffness parameters and best correlated with arterial stiffness variables amongst 24-hour ABPM parameters, especially amongst subjects $<40$ years of age. The pulsatile blood pressure (BP) was a better predictor of aortic PWV than the continuous part of BP.

Categories: Family/General Practice, Internal Medicine

Keywords: arterial stiffness, ambulatory blood pressure monitoring, high blood pressure, pwv, pulse wave velocity

\section{Introduction}

Cardiovascular diseases are the leading cause of death worldwide, and now India is also synchronizing with global data. The major risk factor contributing to cardiovascular diseases is hypertension (HTN), which contributes to nearly 7.1 million premature deaths, two-thirds of all strokes, and half of all myocardial infarction every year [1].

It affected an estimated 118 million inhabitants in India in 2000; this number is projected to double to 214 million by 2025 [2]. HTN is directly responsible for $57 \%$ of all stroke deaths in India and $24 \%$ of all coronary heart disease (CHD) deaths in India [3]. Present estimates suggest that a $2 \mathrm{~mm} \mathrm{Hg}$ population-wide decrease in systolic blood pressure (BP) can lead to the prevention of more than 151,000 strokes and 153,000 CHD deaths in India [4]. 
Recently ambulatory blood pressure monitoring (ABPM) is also gaining importance due to its benefits like measurement of the white coat effect, masked effect, and nocturnal dipping in hypertensives. Arterial stiffness is increasingly recognized as an important and independent contributor to cardiovascular (CV) morbidity and mortality in many patient subsets, particularly in the elderly, hypertensive, and those with end-stage renal disease [5-12]. Aortic stiffening causes loss of elastic recoil and affects the attenuation of the reflected wave, bringing changes in the systolic and diastolic blood pressure (amongst other parameters) in the peripheral and central vessels. Various techniques have been employed for the measurement of arterial stiffness in the central vessels through pulse wave analysis, like pulse wave velocity (PWV) of brachio-ankle and carotid-femoral and augmentation index, of which carotid-femoral PWV (cf-PWV) is a gold standard measure of aortic stiffness $[5,7]$.

Several studies have measured arterial stiffness in Indian subjects, but none has adequately reported its relationship with HTN by employing ambulatory BP monitoring [13-15]. This study was designed to identify the predominant determinant of arterial stiffness as assessed by PWV among different 24-hour ABPM parameters in Indian subjects.

\section{Materials And Methods}

Fifty-one subjects aged between 18 to 60 years presenting to the cardiology outpatient department from a period from January 2017 to December 2017 who were diagnosed to be a case of essential hypertension and who were either on no anti-hypertensive medication or on stable anti-hypertensives for the preceding three months or more were included in the study.

Subjects not willing to participate, diagnosed as a case of secondary hypertension, impaired renal functions, pregnant, with previously established cardiovascular disease, essential hypertension on medication for less than three months, and with a present history of any acute systemic illness were excluded from our study. We also excluded patients with diabetes mellitus or who had a history of tobacco consumption due to potential confounding effects of these factors on arterial stiffness.

The study protocol was approved by the institutional Ethics Committee (IEC). Informed consent was obtained from all the participants prior to their enrollment in the study. Once enrolled, all subjects underwent clinical evaluation and biochemical investigations and assessment of arterial stiffness and ambulatory BP monitoring.

Statistical analyses were done using SPSS for Windows version 24.0 (IBM Inc., Armonk, USA). Comparisons between the groups were carried out using the independent Student t-test. Correlations among different arterial stiffness measurements were assessed using Pearson's correlation coefficients. A p-value $<0.05$ was considered statistically significant.

\section{Results}

The baseline clinical characteristics of the study population are presented in Table 1 . Subjects between the age of 18 to 60 years who were a diagnosed case of essential hypertension $(n=51)$ and who fulfilled the inclusion and exclusion criteria of the study were included in the study. The mean age of all the subjects included in the study was $44.67+10.70$, and $70.6 \%$ of them were males. 


\section{Cureus}

\section{Parameters}

Age (in years)

Gender

Male

Female

Hypertension

Diabetes

Smoker

BMI $\left(\mathrm{kg} / \mathrm{m}^{2}\right)$

Height (cm)

Weight (kg)

Clinic SBP $(\mathrm{mm} \mathrm{Hg})$

Clinic DBP (mm Hg)

Ambulatory BP parameters

24-hour avg. SBP (mm Hg)

24-hour avg. DBP $(\mathrm{mm} \mathrm{Hg})$

Day-time avg. SBP $(\mathrm{mm} \mathrm{Hg})$

Day-time avg. DBP (mm Hg)

Night-time avg. SBP (mm Hg)

Night-time avg. DBP $(\mathrm{mm} \mathrm{Hg})$

24 hr. avg. PP (mm Hg)

Day-time avg. PP (mm Hg)

Night-time avg. PP (mm Hg)

Systolic nocturnal dipping (\%)

Diastolic nocturnal dipping (\%)

Pulse wave velocity parameters

$$
\text { cf-PWV (cm/sec) }
$$

avg. baPWV (cm/sec)

Alx (\%)

Alx (\%) @ HR=75

Heart rate (bpm)

Total cholesterol (mg/dl)

LDL cholesterol (mg/dl)

HDL cholesterol (mg/dl)
Mean +/- SD or $n(\%) ; n=51$

$44.67+10.70$

$36(70.6 \%)$

$15(29.4 \%)$

$51(100 \%)$

$0(0 \%)$

$0(0 \%)$

$28.71+4.65$

$167.57+9.00$

$79.90+15.78$

$142.04+14.41$

$89.57+8.89$

$138.35+13.46$

$86.68+9.33$

$141.55+13.05$

$89.33+9.05$

$130.74+15.54$

$79.05+12.34$

$51.67+9.00$

$52.22+9.13$

$51.70+8.73$

$-7.76+6.04$

$-8.41+6.85$

$1100.98+205.05$

$1564.57+232.12$

$24.41+7.78$

$24.20+9.31$

$75.24+11.44$

$162.78+35.23$

$102.61+29.19$

$41.25+9.60$

\section{TABLE 1: Baseline clinical characteristics of study subjects}

BMI - body mass index; SBP - systolic blood pressure; DBP - diastolic blood pressure; PP - pulse pressure; cf-PWP - carotid to femoral pulse wave velocity; baPWV - brachial-ankle pulse wave velocity; Alx - augmentation index; LDL - low-density lipoprotein; HDL - high-density lipoprotein; avg average; HR - heart rate 


\section{Cureus}

Out of all the subjects ( $\mathrm{n}=51), 29$ subjects $(56.1 \%)$ were on regular treatment with either single or a combination of anti-hypertensive medications for more than three months. The remaining 22 subjects (43.9 \%) were not on any regular anti-hypertensive medication. The distribution of subjects according to BMI is as follows: the majority ( $94.11 \%$ ) were overweight (body-mass index $>23.0 \mathrm{~kg} / \mathrm{m}^{2}$ ) with an overall mean body mass index of $28.71 \mathrm{~kg} / \mathrm{m}^{2}$.

Our study observed that $51 \%$ of subjects had dyslipidemia, out of which only $11.76 \%$ of the total had high total cholesterol, and $45.1 \%$ had low levels of high-density lipoprotein (HDL) cholesterol.

The distribution of 24-hour ambulatory parameters is given in Table 1 .

\section{Arterial stiffness parameters}

We analyzed various pulse wave velocity parameters (cf-PWV, average baPWV, augmentation index [Alx] \%, and $\mathrm{AIx} \%$ at heart rate $[\mathrm{HR}]=75$ ) during the study. We divided the subjects into two groups according to gender and compared the various parameters between the two groups. The results of the independent sample t-test for both males and females are presented in Table 2. Among all the parameters compared between males and females, we found the differences of age, duration of hypertension, 24-hour average pulse pressure, day-time average pulse pressure, night-time average pulse pressure, cf-PWV, and HDL cholesterol to be statistically significant. 


\section{Cureus}

\begin{tabular}{|c|c|c|c|}
\hline Parameters & Male $(n=36)$ & Female $(n=15)$ & $\mathrm{p}$-value \\
\hline Age (years) & $41.53 \pm 10.89$ & $52.2 \pm 5.17$ & 0.001 \\
\hline Duration of hypertension (months) & $41.42 \pm 49.14$ & $87.8 \pm 74.55$ & 0.012 \\
\hline BMI (kg/m2) & $28.62 \pm 4.76$ & $28.91 \pm 4.5$ & 0.843 \\
\hline HR (bpm) & $74.94 \pm 11.44$ & $75.93 \pm 11.79$ & 0.782 \\
\hline Clinic SBP (mm Hg) & $140.83 \pm 12.72$ & $144.93 \pm 18.03$ & 0.360 \\
\hline Clinic DBP (mm Hg) & $90.33 \pm 8.69$ & $87.73 \pm 9.41$ & 0.347 \\
\hline 24-hour avg. SBP (mm Hg) & $136.06 \pm 11.81$ & $143.83 \pm 15.9$ & 0.059 \\
\hline 24-hour avg. DBP (mm Hg) & $86.96 \pm 8.8$ & $86.01 \pm 10.81$ & 0.744 \\
\hline Day-time avg. SBP (mm Hg) & $139.6 \pm 11.45$ & $146.23 \pm 15.73$ & 0.099 \\
\hline Day-time avg. DBP (mm Hg) & $89.85 \pm 8.4$ & $88.09 \pm 10.66$ & 0.531 \\
\hline Night-time avg. SBP (mm Hg) & $128.16 \pm 13.48$ & $136.93 \pm 18.71$ & 0.066 \\
\hline Night-time avg. DBP (mm Hg) & $78.59 \pm 12.05$ & $80.15 \pm 13.39$ & 0.685 \\
\hline 24-hour avg. PP (mm Hg) & $49.1 \pm 7.8$ & $57.83 \pm 8.92$ & 0.001 \\
\hline Day-time avg. PP (mm Hg) & $49.75 \pm 7.84$ & $58.14 \pm 9.53$ & 0.002 \\
\hline Night-time avg. PP (mm Hg) & $49.58 \pm 8.05$ & $56.78 \pm 8.43$ & 0.006 \\
\hline Systolic nocturnal dipping (\%) & $-8.19 \pm 5.96$ & $-6.73 \pm 6.3$ & 0.435 \\
\hline Diastolic nocturnal dipping (\%) & $-9.28 \pm 6.95$ & $-6.33 \pm 6.32$ & 0.163 \\
\hline cf-PWV $(\mathrm{cm} / \mathrm{sec})$ & $1064.15 \pm 195.14$ & $1189.35 \pm 207.60$ & 0.046 \\
\hline avg. baPWV (cm/sec) & $1533.1 \pm 229.2$ & $1640.1 \pm 228.9$ & 0.135 \\
\hline Alx (\%) & $23.75 \pm 6.84$ & $26 \pm 9.75$ & 0.352 \\
\hline Alx (\%) @ HR=75 & $23.5 \pm 8.79$ & $25.87 \pm 10.59$ & 0.414 \\
\hline Total cholesterol (mg/dl) & $160.72 \pm 38.61$ & $167.73 \pm 25.85$ & 0.523 \\
\hline LDL cholesterol (mg/dl) & $102.75 \pm 31.71$ & $102.27 \pm 23$ & 0.958 \\
\hline HDL cholesterol (mg/dl) & $39 \pm 9$ & $46.67 \pm 9.07$ & 0.008 \\
\hline
\end{tabular}

TABLE 2: Results of the independent sample t-test for comparison of the mean value of various parameters between the two groups according to gender

BMI - body mass index; SBP - systolic blood pressure; DBP - diastolic blood pressure; PP - pulse pressure; cf-PWP - carotid to femoral pulse wave velocity; baPWV - brachial-ankle pulse wave velocity; Alx - augmentation index; LDL - low-density lipoprotein; HDL - high-density lipoprotein; avg average; HR - heart rate

We analyzed the subjects into two groups according to age (i.e., age $<40$ years and $>40$ years) and compared the various parameters between the two groups. The results of the independent sample t-test for both the age groups are presented in Table 3. Among all the parameters compared between the two groups, we found the differences between cf-PWV, average baPWV, AIx (\%), AIx (\%) @ HR=75, day-time average pulse pressure, and height to be statistically significant. 


\section{Cureus}

\begin{tabular}{|c|c|c|c|c|c|}
\hline \multirow{3}{*}{ Parameters } & \multicolumn{4}{|c|}{ Age groups } & \multirow{3}{*}{ p-value } \\
\hline & \multicolumn{2}{|c|}{$<40$ years } & \multicolumn{2}{|c|}{$>40$ years } & \\
\hline & Mean & Standard deviation & Mean & Standard deviation & \\
\hline Height (cms) & 175 & 6 & 164 & 8 & $<0.001$ \\
\hline BMI (kg/m2) & 28.6 & 5.1 & 28.8 & 4.5 & 0.895 \\
\hline HR (bpm) & 78 & 11 & 74 & 11 & 0.188 \\
\hline Total cholesterol (mg/dl) & 162 & 35 & 163 & 36 & 0.925 \\
\hline LDL cholesterol (mg/dl) & 105 & 26 & 101 & 31 & 0.661 \\
\hline HDL cholesterol (mg/dl) & 37 & 9 & 43 & 9 & 0.036 \\
\hline cf-PWV (cm/sec) & 972.8 & 125.0 & 1165.0 & 208.4 & 0.001 \\
\hline avg. baPWV (cm/sec) & 1413.7 & 160.4 & 1640.0 & 227.1 & 0.001 \\
\hline Alx (\%) & 20.4 & 6.1 & 26.4 & 7.8 & 0.008 \\
\hline Alx (\%) @ HR=75 & 19.1 & 7.0 & 26.8 & 9.3 & 0.004 \\
\hline 24-hour avg SBP (mm Hg) & 134.0 & 9.9 & 140.5 & 14.6 & 0.103 \\
\hline Day-time avg. SBP (mm Hg) & 137.1 & 10.1 & 143.8 & 13.9 & 0.083 \\
\hline Night-time avg. SBP (mm Hg) & 126.4 & 11.6 & 132.9 & 16.9 & 0.158 \\
\hline 24-hour avg. PP (mm Hg) & 48.7 & 7.5 & 53.5 & 9.3 & 0.069 \\
\hline Day-time avg. PP (mm Hg) & 48.0 & 7.5 & 54.7 & 9.3 & 0.012 \\
\hline Night-time avg. PP (mm Hg) & 50.9 & 8.7 & 52.6 & 9.2 & 0.514 \\
\hline Systolic nocturnal dipping (\%) & -7.7 & 7.1 & -7.8 & 5.5 & 0.956 \\
\hline 24-hour avg. DBP (mm Hg) & 85.92 & 9.47 & 87.05 & 9.38 & 0.688 \\
\hline Day-time avg. DBP (mm Hg) & 89.23 & 9.62 & 89.39 & 8.90 & 0.954 \\
\hline Night-time avg. DBP (mm Hg) & 76.4 & 11.3 & 80.3 & 12.8 & 0.285 \\
\hline
\end{tabular}

TABLE 3: Results of the independent sample t-test for comparison of the mean value of various parameters between the two groups according to age

BMI - body mass index; SBP - systolic blood pressure; DBP - diastolic blood pressure; PP - pulse pressure; cf-PWP - carotid to femoral pulse wave velocity; baPWV - brachial-ankle pulse wave velocity; Alx - augmentation index; LDL - low-density lipoprotein; HDL - high-density lipoprotein; avg average; HR - heart rate

Pearson's correlation coefficients ( $\mathrm{r}$ ) between 24-hour ambulatory blood pressure parameters and pulse wave velocity parameters are presented in Table 4. Bivariate analysis revealed that 24-hour average pulse pressure, day-time average pulse pressure, night-time average pulse pressure, 24-hour average systolic blood pressure (SBP), day-time average SBP, and age were statistically significantly correlated with cf-PWV. Among all the 24-hour ambulatory blood pressure parameters, 24-hour average pulse pressure was observed to have the strongest correlation $(\mathrm{r}=0.414, \mathrm{p}=0.003)$ with arterial stiffness. 


\section{Cureus}

\begin{tabular}{|c|c|c|c|c|c|}
\hline Parameters & & cf-PWV & avg. baPWV & Alx (\%) & Alx (\%) @ HR=75 \\
\hline \multirow{2}{*}{ 24-hour avg. SBP ( $\mathrm{mm} \mathrm{Hg})$} & Pearson correlation & $0.339^{*}$ & $0.324^{*}$ & $0.295^{*}$ & $0.338^{*}$ \\
\hline & $\mathrm{p}$-value & 0.015 & 0.02 & 0.035 & 0.015 \\
\hline \multirow{2}{*}{ 24-hour avg. DBP (mm Hg) } & Pearson Correlation & 0.094 & 0.164 & 0.08 & 0.078 \\
\hline & $\mathrm{p}$-value & 0.512 & 0.249 & 0.576 & 0.588 \\
\hline \multirow{2}{*}{ Day-time avg. SBP (mm Hg) } & Pearson correlation & $0.350^{*}$ & $0.338^{*}$ & $0.317^{*}$ & $0.331^{*}$ \\
\hline & $\mathrm{p}$-value & 0.012 & 0.015 & 0.023 & 0.018 \\
\hline \multirow{2}{*}{ Day-time avg. DBP $(\mathrm{mm} \mathrm{Hg})$} & Pearson correlation & 0.088 & 0.164 & 0.071 & 0.05 \\
\hline & $\mathrm{p}$-value & 0.538 & 0.25 & 0.62 & 0.727 \\
\hline \multirow{2}{*}{ Night-time avg. SBP (mm Hg) } & Pearson correlation & 0.261 & 0.246 & 0.254 & $0.292^{*}$ \\
\hline & $\mathrm{p}$-value & 0.064 & 0.081 & 0.073 & 0.038 \\
\hline \multirow{2}{*}{ Night-time avg. DBP ( $\mathrm{mm} \mathrm{Hg})$} & Pearson correlation & 0.077 & 0.118 & 0.077 & 0.102 \\
\hline & $\mathrm{p}$-value & 0.59 & 0.408 & 0.59 & 0.474 \\
\hline \multirow{2}{*}{ 24-hour avg. PP (mm Hg) } & Pearson correlation & $0.414^{* *}$ & $0.322^{*}$ & $0.372^{* *}$ & $0.425^{* *}$ \\
\hline & $\mathrm{p}$-value & 0.003 & 0.021 & 0.007 & 0.002 \\
\hline \multirow{2}{*}{ Day-time avg. PP (mm Hg) } & Pearson correlation & $0.397^{* *}$ & $0.309^{*}$ & $0.377^{\star *}$ & $0.420^{* *}$ \\
\hline & $\mathrm{p}$-value & 0.004 & 0.027 & 0.006 & 0.002 \\
\hline \multirow{2}{*}{ Night-time avg. PP (mm Hg) } & Pearson correlation & $0.335^{*}$ & 0.195 & $0.288^{*}$ & $0.326^{*}$ \\
\hline & $\mathrm{p}$-value & 0.016 & 0.17 & 0.04 & 0.019 \\
\hline \multirow{2}{*}{ Systolic nocturnal dipping (\%) } & Pearson correlation & -0.012 & -0.03 & 0.021 & 0.069 \\
\hline & p-value & 0.931 & 0.833 & 0.884 & 0.63 \\
\hline
\end{tabular}

\section{TABLE 4: Pearson's correlation coefficient between 24-hour ambulatory blood pressure}

parameters and pulse wave velocity parameters

SBP - systolic blood pressure; DBP - diastolic blood pressure; PP - pulse pressure; cf-PWP - carotid to femoral pulse wave velocity; baPWV brachial-ankle pulse wave velocity; Alx - augmentation index; avg - average; HR - heart rate

** Correlation is significant at the 0.01 level (2-tailed).

* Correlation is significant at the 0.05 level (2-tailed).

We found a statistically non-significant correlation $(\mathrm{r}=-0.012, \mathrm{p}=0.931)$ between systolic nocturnal dipping and cf-PWV. Pearson's correlation coefficient between pulse wave velocity and other clinical parameters is presented in Table 5 . 


\section{Cureus}

\begin{tabular}{|c|c|c|c|c|c|}
\hline \multicolumn{2}{|l|}{ Parameters } & cf-PWV & avg. baPWV & Alx (\%) & Alx (\%)@ HR=75 \\
\hline \multirow{2}{*}{ Age } & Pearson correlation & $0.357^{\star}$ & $0.444^{\star *}$ & $0.330^{*}$ & $0.382^{* *}$ \\
\hline & $p$-value & 0.010 & 0.001 & 0.018 & 0.006 \\
\hline \multirow{2}{*}{ Height (cms) } & Pearson correlation & $-0.356^{*}$ & $-0.348^{*}$ & -0.182 & -0.158 \\
\hline & $\mathrm{p}$-value & 0.010 & 0.012 & 0.202 & 0.269 \\
\hline \multirow{2}{*}{ BMI (kg/m2) } & Pearson correlation & -0.105 & -0.158 & -0.173 & -0.171 \\
\hline & $\mathrm{p}$-value & 0.463 & 0.269 & 0.226 & 0.231 \\
\hline \multirow{2}{*}{ HR (bpm) } & Pearson correlation & -0.079 & -0.089 & -0.069 & $-0.508^{* *}$ \\
\hline & $p$-value & 0.580 & 0.534 & 0.632 & 0.000 \\
\hline \multirow{2}{*}{ Total cholesterol (mg/dl) } & Pearson correlation & 0.113 & 0.155 & 0.018 & 0.083 \\
\hline & $\mathrm{p}$-value & 0.430 & 0.276 & 0.902 & 0.562 \\
\hline \multirow{2}{*}{ LDL cholesterol (mg/dl) } & Pearson correlation & 0.011 & -0.016 & -0.117 & -0.051 \\
\hline & $\mathrm{p}$-value & 0.939 & 0.911 & 0.414 & 0.725 \\
\hline \multirow{2}{*}{ HDL cholesterol (mg/dl) } & Pearson correlation & 0.245 & $0.366^{* *}$ & $0.281^{*}$ & $0.329^{*}$ \\
\hline & p-value & 0.084 & 0.008 & 0.046 & 0.018 \\
\hline
\end{tabular}

\section{TABLE 5: Pearson's correlation coefficient between pulse wave velocity parameters and clinical}

parameters

BMI - body mass index; cf-PWP - carotid to femoral pulse wave velocity; baPWV - brachial-ankle pulse wave velocity; Alx - augmentation index; LDL - low-density lipoprotein; HDL - high-density lipoprotein; avg - average; HR - heart rate

${ }^{\star *}$ Correlation is significant at the 0.01 level (2-tailed).

${ }^{*}$ Correlation is significant at the 0.05 level (2-tailed).

Pearson's correlation coefficient between pulse wave velocity and 24-hour ambulatory blood pressure parameters in the age group $<40$ years and $>40$ years is presented in Table 6 . We found statistically significant correlation in the age group $<40$ years between cf-PWV and both 24 -hour average pulse pressure $(\mathrm{r}=0.54, \mathrm{p}=0.025)$ and night-time pulse pressure $(\mathrm{r}=0.59, \mathrm{p}=0.013)$, respectively. 


\begin{tabular}{|c|c|c|c|c|c|c|c|c|c|}
\hline \multirow[b]{2}{*}{ Parameters } & & \multicolumn{2}{|l|}{ cf-PWV } & \multicolumn{2}{|c|}{ avg. baPWV } & \multicolumn{2}{|l|}{ Alx (\%) } & \multicolumn{2}{|c|}{ Alx (\%) @ HR=75 } \\
\hline & & $\begin{array}{l}\text { Age } \leq 40 \\
\text { years } \\
(n=17)\end{array}$ & $\begin{array}{l}\text { Age }>40 \\
\text { years } \\
(n=34)\end{array}$ & $\begin{array}{l}\text { Age } \leq 40 \\
\text { years } \\
(n=17)\end{array}$ & $\begin{array}{l}\text { Age }>40 \\
\text { years } \\
(\mathrm{n}=34)\end{array}$ & $\begin{array}{l}\text { Age } \leq 40 \\
\text { years }(n=17)\end{array}$ & $\begin{array}{l}\text { Age }>40 \\
\text { years }(n=34)\end{array}$ & $\begin{array}{l}\text { Age } \leq 40 \\
\text { years }(n=17)\end{array}$ & $\begin{array}{l}\text { Age }>40 \\
\text { years }(n=34)\end{array}$ \\
\hline \multirow{2}{*}{ 24-hour avg. SBP } & $\begin{array}{l}\text { Pearson } \\
\text { correlation }\end{array}$ & 0.384 & 0.249 & 0.469 & 0.201 & 0.344 & 0.204 & 0.265 & 0.279 \\
\hline & $\mathrm{p}$-value & 0.128 & 0.155 & 0.057 & 0.255 & 0.177 & 0.247 & 0.304 & 0.110 \\
\hline \multirow{2}{*}{$\begin{array}{l}\text { 24-hour avg. } \\
\text { DBP }\end{array}$} & $\begin{array}{l}\text { Pearson } \\
\text { correlation }\end{array}$ & 0.050 & 0.087 & 0.350 & 0.091 & 0.070 & 0.062 & 0.150 & 0.028 \\
\hline & $\mathrm{p}$-value & 0.850 & 0.626 & 0.168 & 0.609 & 0.791 & 0.729 & 0.565 & 0.876 \\
\hline \multirow{2}{*}{$\begin{array}{l}\text { Day-time avg. } \\
\text { SBP }\end{array}$} & $\begin{array}{l}\text { Pearson } \\
\text { correlation }\end{array}$ & 0.311 & 0.271 & 0.434 & 0.218 & 0.296 & 0.240 & 0.160 & 0.290 \\
\hline & $\mathrm{p}$-value & 0.225 & 0.121 & 0.082 & 0.216 & 0.248 & 0.172 & 0.540 & 0.096 \\
\hline \multirow{2}{*}{$\begin{array}{l}\text { Day-time avg. } \\
\text { DBP }\end{array}$} & $\begin{array}{l}\text { Pearson } \\
\text { correlation }\end{array}$ & -0.009 & 0.131 & 0.334 & 0.129 & 0.048 & 0.085 & 0.085 & 0.038 \\
\hline & $\mathrm{p}$-value & 0.973 & 0.460 & 0.190 & 0.468 & 0.854 & 0.634 & 0.744 & 0.829 \\
\hline \multirow{2}{*}{$\begin{array}{l}\text { Night-time avg. } \\
\text { SBP }\end{array}$} & $\begin{array}{l}\text { Pearson } \\
\text { correlation }\end{array}$ & 0.473 & 0.141 & 0.458 & 0.110 & 0.452 & 0.131 & 0.418 & 0.191 \\
\hline & $\mathrm{p}$-value & 0.055 & 0.426 & 0.064 & 0.534 & 0.069 & 0.459 & 0.095 & 0.278 \\
\hline \multirow{2}{*}{$\begin{array}{l}\text { Night-time avg. } \\
\text { DBP }\end{array}$} & $\begin{array}{l}\text { Pearson } \\
\text { correlation }\end{array}$ & 0.107 & -0.014 & 0.244 & -0.002 & 0.063 & 0.010 & 0.199 & -0.002 \\
\hline & p-value & 0.683 & 0.937 & 0.346 & 0.989 & 0.811 & 0.957 & 0.444 & 0.993 \\
\hline \multirow{2}{*}{ 24-hour avg. PP } & $\begin{array}{l}\text { Pearson } \\
\text { correlation }\end{array}$ & $0.540^{*}$ & 0.303 & 0.284 & 0.224 & 0.472 & 0.259 & 0.209 & $0.410^{*}$ \\
\hline & $\mathrm{p}$-value & 0.025 & 0.081 & 0.270 & 0.203 & 0.056 & 0.139 & 0.420 & 0.016 \\
\hline \multirow[t]{2}{*}{ Day-time avg. PP } & $\begin{array}{l}\text { Pearson } \\
\text { correlation }\end{array}$ & 0.419 & 0.259 & 0.149 & 0.186 & 0.320 & 0.275 & 0.097 & $0.396^{*}$ \\
\hline & $\mathrm{p}$-value & 0.094 & 0.139 & 0.567 & 0.292 & 0.210 & 0.116 & 0.710 & 0.020 \\
\hline \multirow{2}{*}{$\begin{array}{l}\text { Night-time avg. } \\
\text { PP }\end{array}$} & $\begin{array}{l}\text { Pearson } \\
\text { correlation }\end{array}$ & $0.590^{*}$ & 0.265 & 0.163 & 0.177 & 0.421 & 0.222 & 0.279 & 0.332 \\
\hline & $\mathrm{p}$-value & 0.013 & 0.129 & 0.531 & 0.318 & 0.092 & 0.206 & 0.278 & 0.055 \\
\hline \multirow{2}{*}{$\begin{array}{l}\text { Systolic } \\
\text { nocturnal } \\
\text { dipping (\%) }\end{array}$} & $\begin{array}{l}\text { Pearson } \\
\text { correlation }\end{array}$ & 0.267 & -0.114 & 0.130 & -0.102 & 0.252 & -0.084 & 0.331 & -0.035 \\
\hline & p-value & 0.300 & 0.521 & 0.618 & 0.565 & 0.328 & 0.636 & 0.194 & 0.845 \\
\hline
\end{tabular}

TABLE 6: Pearson's correlation coefficient between 24-hour ambulatory blood pressure parameter and pulse wave velocity parameters in different age groups

SBP - systolic blood pressure; DBP - diastolic blood pressure; PP - pulse pressure; cf-PWP - carotid to femoral pulse wave velocity; baPWV brachial-ankle pulse wave velocity; Alx - augmentation index; avg - average; HR - heart rate

${ }^{\star *}$ Correlation is significant at the 0.01 level (2-tailed).

${ }^{*}$ Correlation is significant at the 0.05 level (2-tailed).

\section{Discussion}

Our study is the first study amongst Asian Indians to report the relationship between various 24-hour ambulatory blood pressure parameters and arterial stiffness in middle-aged hypertensive subjects. The 
results of our studies show:

1) arterial stiffness increases progressively as BP increases;

2) 24-hour average pulse pressure, day-time average pulse pressure, night-time average pulse pressure, 24hour average SBP, day-time average SBP, and age were statistically significantly correlated with cf-PWV, the strongest correlation being with 24-hour average pulse pressure;

3) there was no significant correlation between 'nocturnal dipping' and arterial stiffness;

4) in the age group < 40 years, we found a statistically significant correlation between cf-PWV and both 24hour average pulse pressure and night-time pulse pressure;

5) the pulsatile BP had more impact on aortic PWV than the continuous part of BP.

Arterial stiffness is related to BP and is an independent predictor of CV events in hypertensives, so it is recommended in evaluation [16]. Ngim et al. and Stompor et al. reported that cf-PWV was correlated with SBP and also mean arterial pressure (MAP), but not with diastolic blood pressure (DBP) in untreated hypertensive and normotensive middle-aged Malay men and in peritoneal dialysis patients, respectively [16, 17]. Those findings are consistent with our results. Tingli Qin et al. concluded that in patients with grade 1/grade 2 essential hypertension, ambulatory arterial stiffness index (AASI) shows a significant correlation with ambulatory pulse pressure, which is quite similar to our study results [18].

Sa Cunha et al. recommended gender difference - in both sexes, SBP showed a correlation with PWV, while only in women, DBP was correlated with PWV [19]. In contrast, Nurnberger et al. reported that DBP was the only important hemodynamic determinant of PWV in young healthy males [20]. The current study may be confounded by different demographic characteristics such as age range, gender distribution, and BMI than previous studies apart from the smaller sample size. The antihypertensive agents used also might affect the results [21]. Besides, the various methods of BP measurement (e.g., 24-hour ambulatory BP monitoring, casual BP measurement, automatic BP monitoring for 30 minutes) could attribute to the results [22]. Since our findings also revealed the prognostic importance of SBP and PP for aortic PWV and no clear association between DBP and PWV, the results were consistent with previous evidence [23-25]. Aortic PWV has been described as a superior independent indicator of cardiovascular outcome in population-based trials, even after modification of conventional cardiovascular risk factors [26-27]. Elevated PP also has been known to be an independent risk factor of cardiovascular disease [26-30].

Interestingly, Nurnberger et al. reported a contrary result [20]. They showed that among all BP parameters, DBP was the only significant determinant of PWV. But only young (23-35 years of age) healthy males were included in the sample group, where DBP was considered to be the best indicator of coronary heart disease in the Framingham heart study. While the findings of the current study and the Nurnberger study are different, they can jointly reflect the different age-related relationship between BP parameters and PWV. Aortic PWV and BP are proposed to be highly affected by age, and the function of BP parameters as a PWV predictor can vary according to the age spectrum of the population studied.

In a similar study by Kim et al., authors measured BP by invasive measures and also found that among a number of BP parameters, PP demonstrated the greatest association with aortic PWV in normotensive and untreated middle-aged and elderly hypertensive subjects [30]. As the aorta and its first branches are responsible for much of the pathophysiological consequences of arterial stiffness, PWV calculated in the aortic and aortofemoral routes has been recognized to be the most clinically important.

Our study is the first Indian study to report the relationship between various 24-hour ambulatory blood pressure parameters and arterial stiffness in middle-aged hypertensive subjects.

One of the limitations of our study was that although the non-invasive technique showed acceptable reproducibility, the length of the arterial segment was usually estimated by direct superficial measurement of the distance between two transducers. As arteries get longer and more tortuous with age, aortic PWV will therefore be overlooked by a non-invasive procedure. The restricted sample size was also another drawback of the analysis.

\section{Conclusions}

This study concluded that in hypertensive middle-aged subjects, 24-hour average pulse pressure showed the strongest correlation with arterial stiffness and was the predominant determinant of arterial stiffness among different 24-hour ambulatory blood pressure parameters. Pulsatile BP had more impact on aortic PWV than the continuous part of BP. In combination with the previous studies, the present study also suggests that the role of ambulatory BP parameters as a predictor of PWV could be different according to the age range of the population studied. 


\section{Additional Information \\ Disclosures}

Human subjects: Consent was obtained by all participants in this study. Medanta Institutional Ethics Committee issued approval MICR-695/2016. Animal subjects: All authors have confirmed that this study did not involve animal subjects or tissue. Conflicts of interest: In compliance with the ICMJE uniform disclosure form, all authors declare the following: Payment/services info: All authors have declared that no financial support was received from any organization for the submitted work. Financial relationships: All authors have declared that they have no financial relationships at present or within the previous three years with any organizations that might have an interest in the submitted work. Other relationships: All authors have declared that there are no other relationships or activities that could appear to have influenced the submitted work.

\section{References}

1. Kearney P, Whelton M, Reynolds K, et al.: Global burden of hypertension: analysis of worldwide data . Lancet. 2005, 365:217-223. 10.1016/S0140-6736(05)17741-1

2. Gupta R: Trends in hypertension epidemiology in India . J Hum Hyperens. 2004, 18:73-78. 10.1038/sj.jhh.1001633

3. Patel V, Chatterji S, Chisholm D, et al.: Chronic diseases and injuries in India. Lancet. 2011, 377:413-428. 10.1016/S0140-6736(10)61188-9

4. Laurent S, Cockcroft J, van Bortel L, et al.: Expert consensus document on arterial stiffness: methodological issues and clinical applications. Eur Heart J. 2006, 27:2588-2605. 10.1093/eurheartj/ehl254

5. Meaume S, Benetos A, Henry O, Rudnichi A, Safar ME: Aortic pulse wave velocity predicts cardiovascular mortality in subjects $>70$ years of age. Arterioscler Thromb Vasc Biol. 2001, 21:2046-2050. 10.1161/hq1201.100226

6. Sutton-Tyrrell K, Najjar S, Boudreau R, et al.: Elevated aortic pulse wave velocity, a marker of arterial stiffness, predicts cardiovascular events in well functioning older adults. Circulation. 2005, 111:3384-3390. 10.1161/CIRCULATIONAHA.104.483628

7. Boutouyrie P, Tropeano A, Asmar R, et al.: Aortic stiffness is an independent predictor of primary coronary events in hypertensive patients: a longitudinal study. Hypertension. 2002, 39:10-15. 10.1161/hy0102.099031

8. Laurent S, Boutouyrie P, Asmar R, et al.: Aortic stiffness is an independent predictor of all-cause and cardiovascular mortality in hypertensive patients. Hypertension. 2001, 37:1236-1241. 10.1161/01.hyp.37.5.1236

9. Laurent S, Katsahian S, Fassot C, et al.: Aortic stiffness is an independent predictor of fatal stroke in essential hypertension. Stroke. 2003, 34:1203-1206. 10.1161/01.STR.0000065428.03209.64

10. Blacher J, Guerin A, Pannier B, et al.: Impact of aortic stiffness on survival in end-stage renal disease . Circulation. 1999, 99:2434-2439. 10.1161/01.cir.99.18.2434

11. Vlachopoulos C, Aznaouridis K, Stefanadis C: Prediction of cardiovascular events and all-cause mortality with arterial stiffness: a systematic review and meta-analysis. J Am Coll Cardiol. 2010, 55:1318-1327. 10.1016/j.jacc.2009.10.061

12. Milan A, Tosello F, Fabbri A, et al.: Arterial stiffness: from physiology to clinical implications . High Blood Press Cardiovasc Prev. 2011, 18:1-12. 10.2165/11588020-000000000-00000

13. Kasliwal R, Bansal M, Bhargava K, et al.: Carotid intima-media thickness and brachial-ankle pulse wave velocity in patients with and without coronary artery disease. Indian Heart J. 2004, 56:117-122.

14. Mohan V, Gokulakrishnan K, Ganesan A, Kumar SB: Association of Indian diabetes risk score with arterial stiffness in Asian Indian non-diabetic subjects: the Chennai urban rural epidemiology study (CURES-84). J Diabetes Sci Technol. 2010, 4:337-343. 10.1177/193229681000400214

15. Mancia G, De Backer G, Dominiczak A, et al.: 2007 Guidelines for the management of arterial hypertension: The Task Force for the Management of Arterial Hypertension of the European Society of Hypertension (ESH) and of the European Society of Cardiology (ESC). Eur Heart J. 2007, 28:1462-1536. 10.1093/eurheartj/ehm236

16. Ngim C, Rahman A, Ibrahim A: Pulse wave velocity as an index of arterial stiffness: a comparison between newly diagnosed (untreated) hypertensive and normotensive middle-aged Malay men and its relationship with fasting insulin. Acta Cardiol. 1999, 54:277-282.

17. Stompor T, Rajzer M, Sulowicz W, et al.: An association between aortic pulse wave velocity, blood pressure and chronic inflammation in ESRD patients on peritoneal dialysis. Int J Artif Organs. 2003, 26:188-195. 10.1177/039139880302600302

18. Qin T, Jiang H, Jiao Y, et al.: Ambulatory arterial stiffness index correlates with ambulatory pulse pressure but not dipping status in patients with grade 1/grade 2 essential hypertension. J Int Med Res. 2014, 13231334. 10.1177/0300060514548288

19. Sa Cunha R, Pannier B, Benetos A: Association between high heart rate and high arterial rigidity in normotensive and hypertensive subjects. J Hypertens. 1997, 15:1423-1430. 10.1097/00004872-19971512000009

20. Nurnberger J, Dammer S, Opazo Saez A, Philipp T, Schäfers RF: Diastolic blood pressure is an important determinant of augmentation index and pulse wave velocity in young, healthy males. J Hum Hypertens. 2003, 17:153-158. 10.1038/sj.jhh.1001526

21. Asmar R, Brunel P, Pannier B, Lacolley PJ, Safar ME: Arterial distensibility and ambulatory blood pressure monitoring in essential hypertension. Am J Cardiol. 1988, 61:1066-1070.

22. Franklin S, Gustin W, Wong N: Hemodynamic patterns of age-related changes in blood pressure. The Framingham heart study. Circulation. 1997, 96:308-315. 10.1161/01.cir.96.1.308

23. Khattar R, Swales J, Dore C, Senior R, Lahiri A: Effect of aging on the prognostic significance of ambulatory systolic, diastolic, and pulse pressure in essential hypertension. Circulation. 2001, 104:783-789. 


\section{Cureus}

10.1161/hc3201.094227

24. Franklin S, Larson M, Khan S, et al.: Does the relation of blood pressure to coronary heart disease risk change with aging? The Framingham heart study. Circulation. 2001, 103:1245-1249.

10.1161/01.cir.103.9.1245

25. Amar J, Ruidavets J, Chamontin B, Drouet L, Ferrières J: Arterial stiffness and cardiovascular risk factors in a population-based study. J Hypertens. 2001, 19:381-387. 10.1097/00004872-200103000-00005

26. Willum-Hansen T, Staessen J, Torp-Pedersen C, et al.: Prognostic value of aortic pulse wave velocity as index of arterial stiffness in the general population. Circulation. 2006, 113:664-670.

10.1161/CIRCULATIONAHA.105.579342

27. de Simone G, Roman M, Koren M, et al.: Stroke volume/pulse pressure ratio and cardiovascular risk in arterial hypertension. Hypertension. 1999, 33:800-805. 10.1161/01.HYP.33.3.800

28. Benetos A, Safar M, Rudnichi A, et al.: Pulse pressure: a predictor of long-term cardiovascular mortality in a French male population. Hypertension. 1997, 30:1410-1415. 10.1161/01.HYP.30.6.1410

29. Verdecchia P, Schillaci G, Borgioni C, et al.: Ambulatory pulse pressure: a potent predictor of total cardiovascular risk in hypertension. Hypertension. 1998, 32:983-988. 10.1161/01.hyp.32.6.983

30. Kim E, Park C, Park J, et al.: Relationship between blood pressure parameters and pulse wave velocity in normotensive and hypertensive subjects: invasive study. J Hum Hypertens. 2007, 21:141-148. 10.1038/sj.jhh.1002120 